\title{
Research on RF Tag Performance on Metal Media and Design of Slotting Tag
}

\author{
Yanghua Gao ${ }^{1}$ and Zhihua Zhang ${ }^{2}$ \\ ${ }^{1}$ Information Center, China Tobacco Zhejiang Industrial Co., Ltd \\ Hangzhou \\ ${ }^{2}$ Information Center, China Tobacco Zhejiang Industrial Co., Ltd \\ Hangzhou \\ yhgao@zju.edu.cn,zzh@zjtobacco.com
}

\begin{abstract}
Passive radio frequency $(R F)$ tags in the $U H F$ and microwave bands have recently drawn considerable attention with their great potential for use in inventory management, parcel and postal tracking. However, much more basic research is needed to increase the range and reliability of a passive $R F$ tag's radio link, particularly when the $R F$ tag is placed onto a lossy dielectric object or a metallic surface. This paper presents two new useful forms of the radio link budget that describe the power link of an RF tag system when the tag is attached to an object, then the effects of a metal plate to antenna parameters when the antenna is horizontally placed near the plate. Furthermore, according to a series of tests for measuring the performance of an RF tag, relationship between distance from tag to a sheet of copper surface and readability is discovered. Moreover, the magnifying effect of an extra metal layer to readability is found too. Finally, according to the results of experiments, a design method of slotting RF tag which can be used to identify the goods location is proposed. This method can not only eliminate negative effect of metal disturbance, but enhance readability, which considers the convenience for use and manufacture cost as well.
\end{abstract}

Keywords: RFID, Link budget, Mental media, slotting tag

\section{Introduction}

Passive modulated backscatter RF tags are transponders that communicate with an interrogator, or reader, using far-field electromagnetic waves. These tags operate in the UHF and microwave frequency bands and typically modulate the backscattered radiation reflected from the tag antenna using load modulation. In recent years, the use of passive RF tags for radio frequency identification (RFID) has generated increasing interest because of their potential applications to inventory management, parcel and postal tracking, access control, airport luggage, self check-out, and medical ID bracelets [1-6]. RFID applications of RF tags are expected to increase dramatically in coming years due to mandates issued by Wal-Mart and the Department of Defense for their suppliers to begin using RFID tags on their shipments [7-11]. Although these entities desire to label and track individual items in their inventory, the relative high cost and poor performance of passive RF tags limit their use to tracking at the pallet or crate level [12-19], especially, disturbance of metal media on radio frequency signal is always influencing the effective application of RFID. However, very few papers provided an overview of the principle of triggering this disturbance and the methods. In 
this paper, we attempted to fill the gap. We explored the effects of a metal plate to antenna parameters. Furthermore, the performance of an RF tag is analyzed and discussed according to a series of tests. Finally, a design method of slotting RF tag is proposed based on the research.

\section{Backscatter RFID System}

The operation of a typical passive backscatter RFID system includes an RFID reader and a passive RFID tag, shown as Figure 1 [16-20]. A passive tag consists of an antenna and an application specific integrated circuit chip, both with complex impedance $\left(Z_{\text {in }}\right.$ and $\left.Z_{L}\right)$. The chip obtains power and data from the radio frequency (RF) signal transmitted by the RFID reader. The tag sends data back by switching its input impedance between two states $\left(Z_{c l}\right.$ and $Z_{c 2}$ ) and thus modulating the backscattered signal. At each impedance state, the tag presents a certain radar cross-section (RCS). One of the impedance states is generally high $\left(\mathrm{RCS}_{1}\right)$ and another is low $\left(\mathrm{RCS}_{2}\right)$ to provide a significant difference in the backscattered signal.

The variation of the chip impedance with power and frequency can drastically affect the performance of the tag. Thus, proper impedance match between the antenna and the chip is very important in passive RFID systems. It directly influences RFID system performance characteristics such as the reading ability. Usually, in order to maximize the reading ability, the antenna impedance is matched to the chip impedance at the minimum power level required for the chip to work.

Data exchange between reader and tag can employ various modulation and coding schemes. The signal transmitted on the uplink (reader to the tag) contains both continuous wave $(\mathrm{CW})$ and modulated commands as shown in Figure 1. On the downlink (tag to the reader), the data is sent back during one of $\mathrm{CW}$ periods when the tag impedance modulates the backscattered signal $[6,7,10,11,18]$. A passive backscatter RFID system can be produced at a favorable price, but the read ability is strongly limited by the overall efficiency of the system. The antenna characteristics have a radical effect on the read ability of RFID systems. In the following paragraphs, we study the response model of passive backscatter RFID systems and the calculation of the read distance.

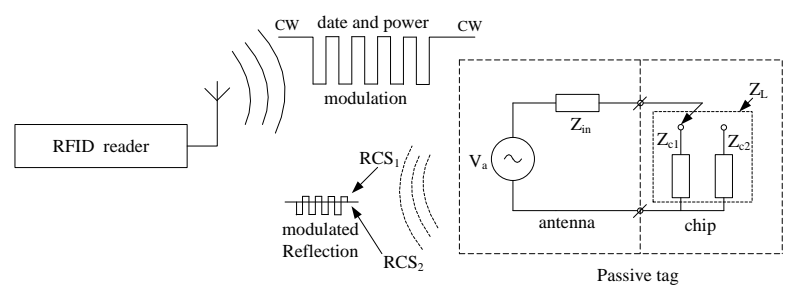

Figure 1. Passive backscatter RFID system

\section{Radio Link Budgets}

Equation (1) is the power radio link budget that describes the amount of power available to the RF tag for operation (uplink) [15-16]. It is a modification of the Friis transmission equation and assumes that the antennas have an impedance and polarization match.

$$
P_{\text {tag }}=P_{\text {reader }-t}-L_{s}+G_{\text {reader }-t}+G_{\text {tag }}-G_{p}-20 \log _{10}\left(\frac{4 \pi}{\lambda}\right)-20 \log _{10}(d)
$$


Where $P_{t a g}$ - power at the RF tag antenna terminals;

$P_{\text {reader-t }}-$ power input to the reader transmit antenna;

$L_{s}{ }^{-}$system losses in both the tag and reader;

$G_{\text {reader-t }}$ - gain of the reader transmit antenna;

$G_{t a g}-$ RF tag antenna gain in free space;

$G_{p}$ - gain penalty due to material attachment;

$20 \log _{10}\left(\frac{4 \pi}{\lambda}\right)$ - a loss dependent upon the free space wavelength;

$20 \log _{10}(d)$ - free space path loss referenced to $1 \mathrm{~m}$.

The backscatter communication radio link budget, a modification of the monostatic radar is shown in equation (2), describes the amount of modulated power that is scattered from the RF tag to the tag reader (downlink):

$$
P_{\text {reader }-r}=P_{\text {reader }-1}-L_{s}+G_{\text {reader }-}+G_{\text {reader }- \text { r }}+10 \log _{10}\left(\frac{\sigma}{4 \pi}\right)-20 \log _{10}\left(\frac{4 \pi}{\lambda}\right)-40 \log _{10}(d)
$$

Where $P_{\text {reader-r }}$ - power at the reader receive antenna;

$P_{\text {reader- } t}-$ power input to the reader transmit antenna;

$G_{\text {reader- }-}$ - gain of the reader transmit antenna;

$G_{\text {reader-r }}$ - gain of the reader receive antenna;

$\sigma$ - radar cross section of the RF tag.

All other terms are de fined in (1) and all antennas are assumed to have an impedance and polarization match. The radar cross section (RCS) of the RF tag is primarily due to scattering by the RF tag antenna and can be written as the sum of a structural mode and an antenna mode [6]. The scattered fields due to the structural mode are radiated by currents induced by the incident field on the surface of the antenna. The structural mode is not dependent upon the RF tag antenna load and therefore, power scattered due to this term is not modulated [17-24].

\section{Calculation of the Read Distance}

Assuming that the threshold power at the RF tag antenna terminals is $P_{t \min }$ and the threshold power at the reader receive antenna is $P_{r \min }$, according to radio link budges, when $P_{\text {tag }} \geq P_{t \text { min }}$ and $P_{\text {reader }-r} \geq P_{r \text { min }}$, the RFID system can be operated.

When

$$
P_{\text {tag }}=P_{t \min }
$$

Then

$$
P_{t \min }=\frac{P_{\text {reader }-t} G_{\text {reader }-t} G_{\text {tag }} \lambda^{2}}{(4 \pi)^{2} R_{t}^{2}}
$$


The maximum read distance of uplink can be obtained as

$$
R_{t \max }=\frac{\lambda}{4 \pi} \sqrt{\frac{P_{\text {reader }-t} G_{\text {reader }-t} G_{\text {tag }}}{P_{t \min }}}
$$

In free place

$$
S_{i}=\frac{E^{2}}{2 \mu_{0}}
$$

Where $\mu_{0}=120 \pi$

Then

$$
R_{t \max }=\frac{\sqrt{60 P_{\text {reader }-t} G_{\text {reader }-t}}}{E_{0}}
$$

When

$$
P_{\text {reader }-r}=P_{r \text { min }}
$$

Then the maximum read distance of downlink in free space can be expressed as

$$
R_{r \max }=\frac{\lambda}{4 \pi}\left[\frac{P_{\text {reader }-t} G_{\text {reader }-t}^{2} G_{\text {read }-r}}{\mathrm{P}_{r \min }}\right]^{\frac{1}{4}}
$$

When the passive backscatter RFID system works, the maximum read distance of the system can be expressed as

$$
R_{\max }=\min \left\{R_{t \max }, R_{r \max }\right\}
$$

\section{RF Tag Performance on Various Materials}

Table1 shows the average RF tag antenna gain penalties. These values were calculated by linearly averaging gain penalty measurements for material. As Table 1 shows, the average gain penalty increases with the increasing loss tangent of each material. The individual measurements showed high precision for the dielectric materials with a small loss tangent (i.e. cardboard and water). However, for the highly conductive materials, the measured gain penalty for each antenna was varied respectively. The values from Table 1 in conjunction with equations (1) and (2) show that the decrease in antenna performance due to material loss can be significant. For the backscatter link (downlink), the measurements show that an antenna attached to metal will decrease the tag antenna gain by $9.7 \mathrm{~dB}$.

\section{Table1. RF tag performance on various materials}

\begin{tabular}{|l|l|l|l|l|l|}
\hline & Cardboard & Wood & Water & Beef & Metal \\
\hline $\begin{array}{l}\text { Average Gain } \\
\text { Penalty (dB) }\end{array}$ & 0.7 & 4.9 & 5.6 & 7.7 & 9.7 \\
\hline
\end{tabular}




\section{Effects of a Metal Plate to Antenna Parameters}

In passive backscatter RFID systems, the receiving power of antenna is related to power transmission coefficient $(\tau)$, in free space, $\tau=1$. Assuming that the antenna impedance is $Z_{\mathrm{c}}=R_{\mathrm{c}}+j X_{\mathrm{c}}$ and the chip impedance $Z_{\mathrm{in}}=R_{\mathrm{in}}+j X_{\mathrm{in}}$, can be expressed as

$$
\tau=\frac{4 R_{i n} R_{L}}{\left|Z_{\text {in }}+Z_{L}\right|^{2}} \quad(0 \leq \tau \leq 1)
$$

According to the definition of the gain of tag antenna, the gain of tag antenna can be expressed as

$$
G_{r}=D_{r} e_{r}
$$

Where $D_{\mathrm{r}}$ is the direction of tag antenna, $e_{\mathrm{r}}$ is radiant efficiency.

The transmitted power of reader, gain of reader antenna and the sensitivity of the tag chip are unchangeable in the same passive backscatter RFID system. Thus the read rate is related to transmission coefficient, the direction of tag antenna and radiant efficiency.

\subsection{Effects of boundary of metal media}

The read rate is lower because the phase is reverse between transmitted signal of the reader and its echo wave when the RF tag is placed on metal media. The electric field ponderance is shown as Figure 2 when the relation is vertical between the incidence wave and metal surface. From Figure 2, the electric field power is minimum when the distance between tag and metal media is zero, while the power is maximum when the distance is $0.25 \lambda$.

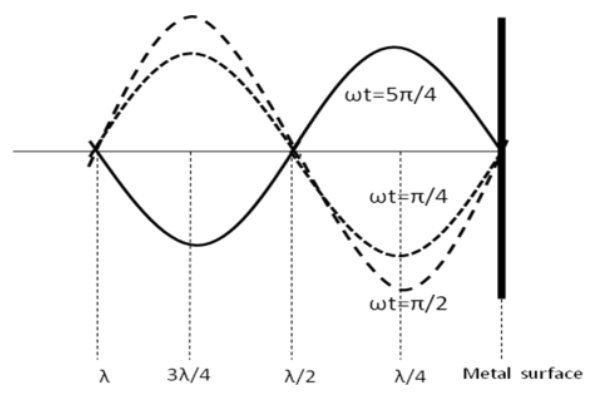

Figure 2. The distributing mode of electric field of metal surface

The distance between dipole antenna and metal media is related to gain of antenna, which is shown as Figure 3. The gain is near zero when the distance is $\lambda / 2$.

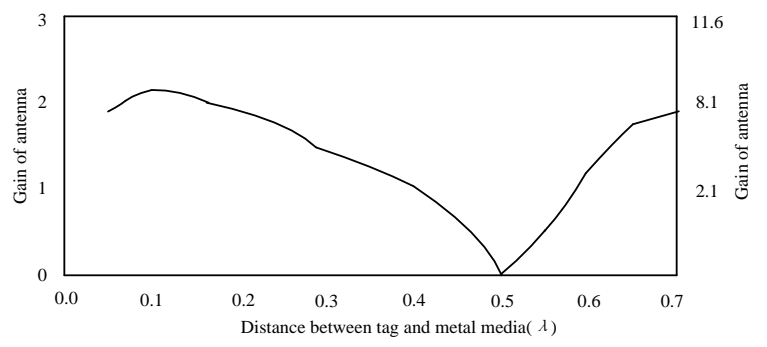

Figure 3. Relationship between distance and gain of antenna 
FEM(Finite Element Method) has been used to simulate dependency of antenna parameters on the six of the distance between the metal media and the tag antenna, which are shown as Figure 4(where $D$ is the distance between metal media and tag antenna). From Figure 4 , when the distance is $\lambda / 32$ or $\lambda / 4$, assuming that the gain of antenna is $G$ and $G_{r}$ is the gain of antenna during in free space, then

$G>G_{r}$

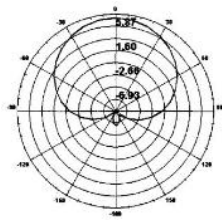

(a) $\mathrm{D}=\lambda / 32$

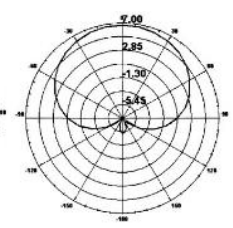

(b) $\mathrm{D}=\lambda / 4$

\section{Figure 4. Relationship between the gain mode of dipole antenna and the distance of metal media}

\subsection{Effects of eddy current of metal media}

In passive RFID backscatter systems, the read rate will be lower if the tag is placed near the metal media because of the eddy current which consumes the power from the reader. Furthermore, the extral magnetic field is created by the eddy current which will make the electromagnetism field of frequency to distort. The effects can be shown as Figure 5. The read rate will be improved if the phase of echo magnetic field from the metal media is the same as the magnetic of the reader, thus the extra metal is needed to create the second echo magnetic field.

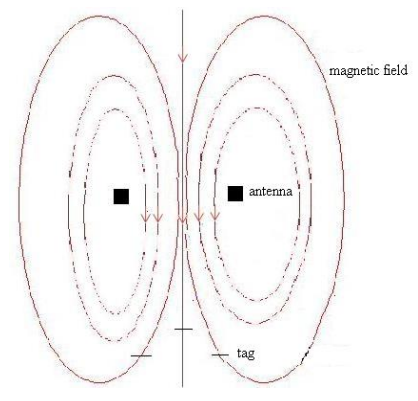

(a) The RF tag without metal media

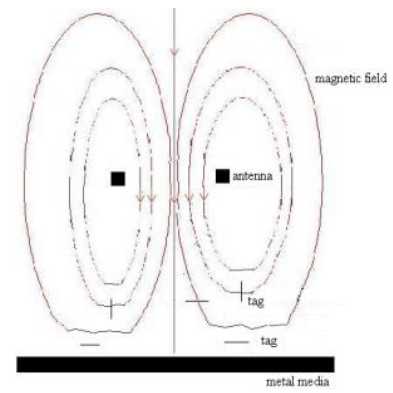

(b) The RF tag with metal media

Figure 5. The effects of metal media

\subsection{Radio assay tests}

The test RF system is designed to measure the RF tag performance through a series of tests, which includes a RF tag and portable RF reader. The work frequency of the test system is $915 \mathrm{MHZ}$ and the protocol is $I S O-18000-6 \mathrm{~B}$, furthermore, the read distance of the system is $1 \mathrm{~m}$.

Test1: When the RF tag is attached to a sheet of copper (the thickness of copper is $1 \mathrm{~mm}$ ), the read rate is measured. The results are shown as Table 2. The read rate is near zero as respected. 
Test2: When the distance between RF tag and the sheet of copper is $10 \mathrm{~mm}$, the read rate is measured.

Test3: When the distance between RF tag and the sheet of copper is various, the read rate is measured.

Test4: When an extra metal media (a sheet of copper) is used to create the second echo magnetic field and the distance between RF tag and the sheet of copper is various, the read rate is measured.

Test5: When the sheet of copper is taken off, the read rate is measured.

Table 2. Radio assay results

\begin{tabular}{|c|c|c|c|c|c|c|c|c|}
\hline \multirow[b]{2}{*}{ Varrous sthuation } & \multirow{2}{*}{$\begin{array}{c}\text { distance } \\
(m m)\end{array}$} & \multicolumn{6}{|c|}{ Read rate (times/second) } & \multirow{2}{*}{$\begin{array}{l}\text { Average } \\
\text { read rate } \\
\text { (ttmess) })\end{array}$} \\
\hline & & $\begin{array}{l}\text { First } \\
\text { time }\end{array}$ & $\begin{array}{l}\text { Second } \\
\text { time }\end{array}$ & $\begin{array}{l}\text { Third } \\
\text { time }\end{array}$ & $\begin{array}{l}\text { Fourth } \\
\text { timme }\end{array}$ & $\begin{array}{l}\text { Fifh } \\
\text { time }\end{array}$ & $\begin{array}{l}\text { Sixth } \\
\text { time }\end{array}$ & \\
\hline copper RF tag $^{2}$ & 0 & 0.2 & 0.2 & 0.1 & 0.1 & 0.1 & 0.1 & 0.13 \\
\hline coppet $\| \mathrm{RF}_{\mathrm{fag}}$ & 10 & 6.3 & 6.2 & 6.1 & 6.1 & 6.1 & 6.2 & 6.17 \\
\hline \multirow{12}{*}{ copper $\left.\right|_{R F} ^{\text {fogm }}$} & 1 & 0.2 & 0.2 & 0.2 & 0.3 & 0.1 & 0.2 & 0.20 \\
\hline & 2 & 1.4 & 1.7 & 1.4 & 1.5 & 1.7 & 1.6 & 1.55 \\
\hline & 3 & 2.7 & 2.8 & 3.0 & 3.0 & 2.8 & 2.8 & 2.86 \\
\hline & 4 & 3.6 & 3.8 & 3.6 & 4.0 & 3.7 & 4.1 & 3.80 \\
\hline & 5 & 4.7 & 4.4 & 4.5 & 4.5 & 4.8 & 4.2 & 4.52 \\
\hline & 6 & 5.4 & 5.6 & 5.6 & 5.8 & 5.7 & 5.5 & 5.60 \\
\hline & 7 & 6.5 & 6.2 & 6.8 & 6.5 & 6.4 & 6.4 & 6.47 \\
\hline & 8 & 6.7 & 7.0 & 6.5 & 6.9 & 7.0 & 7.2 & 6.88 \\
\hline & 9 & 7.0 & 7.5 & 6.8 & 7.4 & 7.4 & 7.5 & 7.27 \\
\hline & 10 & 7.2 & 6.9 & 7.0 & 7.5 & 7.5 & 7.2 & 7.22 \\
\hline & 11 & 7.5 & 7.7 & 7.0 & 6.9 & 7.5 & 7.6 & 7.37 \\
\hline & 12 & 7.2 & 7.4 & 7.6 & 7.4 & 7.3 & 7.2 & 7.35 \\
\hline \multirow{9}{*}{ copper $\sum_{R F \text { toe }}^{\text {foum }}$} & 4 & 3.4 & 3.2 & 3.7 & 3.4 & 3.4 & 3.0 & 3.35 \\
\hline & 5 & 4.5 & 4.8 & 5.0 & 4.8 & 4.6 & 4.6 & 4.72 \\
\hline & 6 & 5.9 & 6.2 & 6.0 & 5.8 & 6.0 & 6.0 & 5.98 \\
\hline & 7 & 6.8 & 7.0 & 7.2 & 6.8 & 7.2 & 7.0 & 7.00 \\
\hline & 8 & 9.2 & 9.7 & 9.4 & 9.4 & 9.6 & 9.4 & 9.45 \\
\hline & 9 & 10.5 & 10.0 & 11.0 & 10.4 & 11.2 & 10.8 & 10.65 \\
\hline & 10 & 11.2 & 11.8 & 12.0. & 12.4 & 11.4 & 11.4 & 11.70 \\
\hline & 11 & 12.4 & 12.8 & 12.0 & 13.5 & 13.2 & 13.0 & 12.82 \\
\hline & 12 & 13.1 & 13.4 & 12.9 & 13.8 & 13.4 & 13.2 & 13.30 \\
\hline \RF tag & $\begin{array}{l}\text { Without } \\
\text { copper }\end{array}$ & 7.9 & 8.4 & 8.7 & 8.0 & 8.4 & 8.5 & 8.32 \\
\hline
\end{tabular}

\subsection{Analysis of tests results}

From Table 2, the read rate is improved with the distance increasing, which is accord with research results. Furthermore, the effect of extra magnetic field is obvious. The tests results of test 3 and test 4 are shown as Figure 6. From Figure 6, the read rate can be improved by the method such as test 4 where the tests result is better than test 5 . 


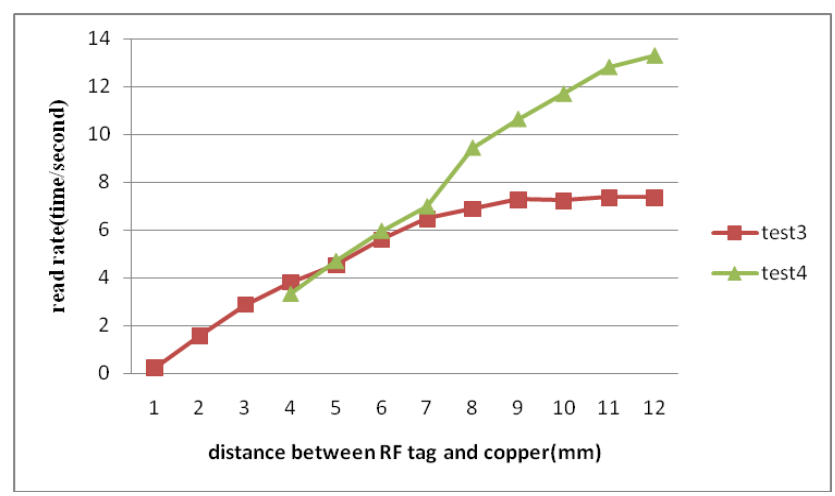

Figure 6. Radio assay results

\section{Design of Slotting RF Tag}

\subsection{Slotting RF tag}

The slotting RF tag is designed in order to realize the warehouse digital management where the minimum management cell is goods location. The slotting RF tag includes five parts. The first is passive RF tag, the second is partition composed of foam, the third is a sheet of copper which can create extra echo magnetic field to improve read rate, the fourth is underlay which can protect the passive RF tag, the last is also a sheet of copper which create the first echo magnetic field from the RF reader. The structure of the slotting RF tag is shown as Figure 7.

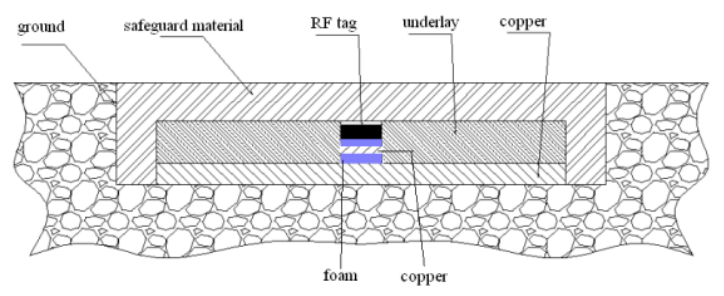

Figure 7. The structure of slotting RF tag

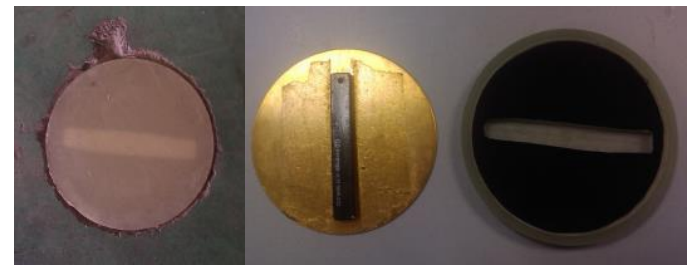

Figure 8. Actual picture of slotting tag

\subsection{Application of slotting tag}

The application system of slotting tag includes location tag, pallet with passive RF tag and forklift with RFID reader, which is shown as Figure 9. 


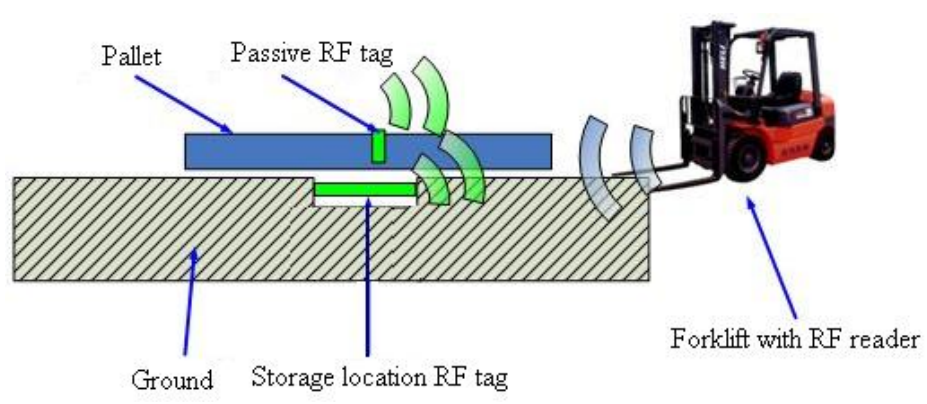

Figure 9. Application system

The pallets with passive tag are used to carry goods and the information of bar code is written in the passive RF tag, which can ensure synchronous operation of practicality stream and information stream. The slotting tag is the identification of goods location which includes the information of the location. The forklift with RF reader is used to carry goods and read the information of bar code which has been written in pallet RF tag.

\subsection{Operation Flow of Warehousing Management Based on Slotting Tag}

The operation flow of warehouse management includes warehouse entry, picking and delivery.

7.3.1. Flow of warehouse entry: (1) After receiving the entry order from the ERP system, the warehouse management system will dispatch each individual pallet upon which thirty boxes of goods are fixed, and generate receiving order according to the operation requirements.

(2) The warehouse management system will generate the warehousing entry, search idle forklifts through the wireless network, then the entry order to the front-end system (including forklift, mobile terminal and RFID reader).

(3) When the front-end system receives the warehousing entry, the driver will drive the forklift to transport the pallet to appointed position and read the pallet tags which include the goods information by the RFID reader, then sent it to warehouse management system.

(4) The front-end system will send the confirmed data to the warehouse management system automatically. After obtaining the data, the warehouse management system will update the correspondent system data, indicate the current position of pallet, and close the entry order. The warehouse entry flow is shown in Figure 10. 


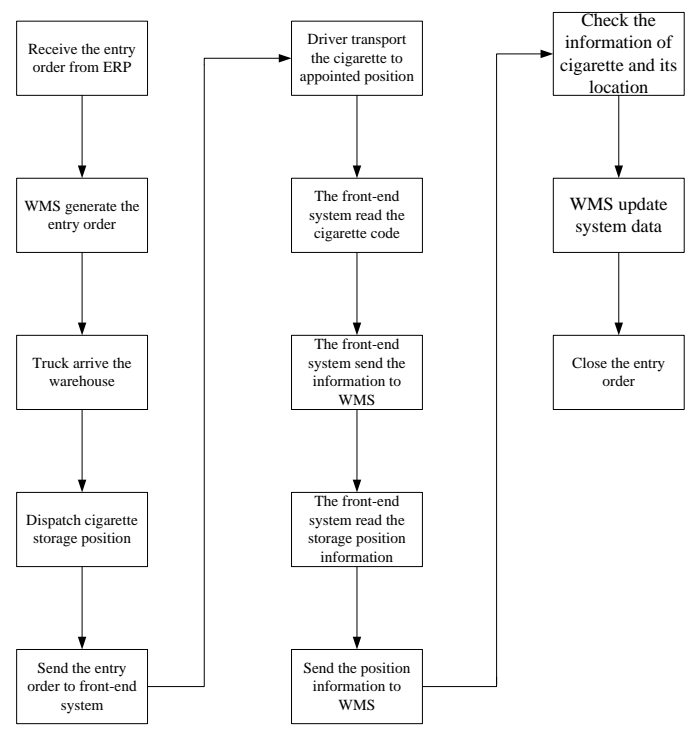

Figure 10. Flow of warehouse entry

7.3.2. Flow of Picking Operation: (1) The warehouse management system will generate picking orders according to requirements.

(2) The warehouse management system will send picking operation order to the frontend system through the wireless network.

(3) The front-end system will receive the picking order. The driver reads pallet tags to obtain the information of the goods location through RFID reader, and sends it to the warehouse management system.

(4) The front-end system checks whether the data collected are matched with the system order or not. If confirmed, the driver will transport the goods on the pallet out of the warehousing position.

(5) The RFID system will update the data in the tags of goods location in the warehousing position. The front-end system will send the results to the warehouse management system through the wireless network, and the warehouse management system will update the correspondent data in the system.

7.3.3. Flow of Goods Delivery: (1) The warehouse management system will generate the withdrawing order according to requirements, and then send the operation order to the front-end system through the wireless network.

(2) After the front-end system receives the withdrawing order, the warehouse management system will obtain the cigarette information when appointed forklifts are driven through RFID readers fixed on the forklifts, send the detailed list of cigarette to be delivered to the mobile terminal, displaying to the driver.

(3) The front-end system reads the position tags, and sends it to warehouse management system.

(4) The warehouse management system will check whether the data collected are matched with the system order. If confirmed, the system will direct the driver to transport the cigarette to the appointed delivery area. 
(5) After completing the assignment, the front-end system will send the data confirmed to the warehouse management system, and then the latter will update the system data, and close the delivery order. The warehouse delivery flow is shown in Figure 11.

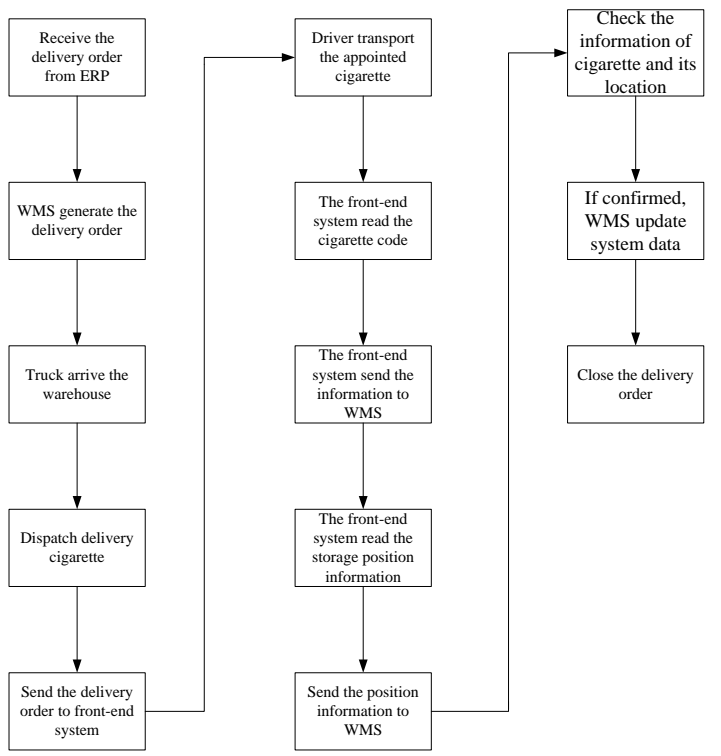

Figure 11. Flow of goods delivery

\section{Conclusion}

This paper explored the principle of triggering the disturbance of metal media on RF tag. Furthermore, a series of assay to measure the performance of passive RF attached to metal media was presented. The read rate increased with the increasing distance between RF tag and the metal media. The read rate of passive backscatter RF system can be improved by the extra sheet copper which is used to create the second echo magnetic field. Finally, according to the results of radio assay, a design method of slotting RF tag which can be used in digital warehouse management was proposed. This method can not only eliminate negative effect of metal disturbance, but enhance readability.

Where there is great love, there are always miracles. Love is like a butterfly. It goes where it pleases and it pleases where it goes. If I had a single flower for every time I think about you, I could walk forever in my garden. Within you I lose myself, without you I find myself wanting to be lost again. At the touch of love everyone becomes a poet.

\section{References}

[1] K. V. S. Rao, "An Overview of Backscattered Radio Frequency Identification System (RFID)”, 1999 Asia Pacific Microwave Conference, vol. 3, (1999), pp. 746-749.

[2] I. D. Robertson and I. Jalaly, "RF ID Tagging Explained", Communications Engineer, vol. 1, no. 1, (2003), pp. 20-23.

[3] K. Finkenzeller, "RFID Handbook: Fundamentals and Applications in Contactless Smart Cards and Identification", John Wiley and Son LTD, New York, 2nd edition, (2003).

[4] T. Purdum, "Factory to Foxhole: RFID Deadline Looms", Industry Week, vol. 253, no. 11, (2004), pp. 12.

[5] J. D. Griffin, "RF tag Antenna Performance on Various materials Using Radio Link Budgets", IEEE Antennas and Wireless Propagation Letters, vol. 5, (2006), pp. 247-250. 
[6] Z. Tang, "The Effects of Antenna Properties on read Distance in Passive backscatter RFID Systems", 2009 Int'l Conf. on Networks Security, Wireless Comm. and Trusted Computing, vol. 1, (2009), pp. 120-123.

[7] Y. Gao, "Research on Supply Chain Optimal Control Based on RFID Integrated System", IEEE 2012 Int'1 Conf. on Information Mgt, Innovation Management and Industrial Engineering, vol. 1, (2012), pp. 407-409.

[8] J. D. Griffin, "RF Tag Antenna Performance on Various Materials Using Radio Link Budgets", IEEE antennas and wireless propagation letters, vol. 5, (2006), pp. 247-248.

[9] F. TongKe, "Smart Agriculture Based on Cloud Computing and IOT", JCIT: Journal of Convergence Information Technology, vol. 8, no. 2, (2013), pp. $210 \sim 216$.

[10] F. Feng and X. Qi, "Locating Method in the Warehouse Based on Active RFID Tags", JDCTA: International Journal of Digital Content Technology and its Applications, vol. 7, no. 1, (2013), pp. $223 \sim 231$.

[11] Y. S. Mezaal, "A New Microstrip Bandpass Filter Design Based on Hilbert Fractal Geometry for Modern Wireless Communication Applications", IJACT: International Journal of Advancements in Computing Technology, vol. 1, no. 2, (2009), pp. $35 \sim 39$.

[12] J. Lee, J. Song, K. Oh and N. Gu, "Information lifecycle management with RFID for material control on construction sites", Advanced Engineering Informatics, vol. 27, no. 1, (2013), pp. 108-119.

[13] K. Domdouzis, B. Kumar and C. Anumba, "Radio-frequency identification (RFID) applications: a brief introduction", Advanced Engineering Informatics, vol. 21, no. 4, (2007), pp. 350-355.

[14] D. Henrici, "Security and Privacy in large-scale RFID system: challenges and solutions", (PhD dissertation), University of Kaiserslautern, (2008).

[15] G. Guven, G. Demiralp and E. Ergen, "Value of RFID Technology in Construction Supply Chains", In the Value of RFID, Springer London, (2013), pp. 111-127.

[16] H. Yan and H. Hu, "Research and realization on CDIO teaching experimental system based on RFID technique of internet of things", 2011 International Conference on Mechatronic Science, Electric Engineering and Computer. IEEE, (2011), pp. 841-844.

[17] H. Hamalainen and J. Ikonen, "Requirements for RFID tagging process of concrete elements in building project, Software”, 16th International Conference on Telecommunications and Computer Networks, IEEE, (2008), pp. 238-242.

[18] C. P. Cheng and Y. H. Ma, "RFID Supported Cooperation for Construction Waste Management", 8th International Conference on Cooperative Design, Visualization, and Engineering, (2011), pp. 125-128.

[19] S. N. Razavi and C. T. Haas, "Using reference RFID tags for calibrating the estimated locations of construction materials", Automation in Construction, vol. 20, no. 6, (2011), pp. 677-685.

[20] J. M. Sardroud, "Influence of RFID technology on automated management of construction materials and components", Scientia Iranica, vol. 19, no. 3, (2012), pp. 381-392.

[21] G. Demiralp and G. E. Ergen, "Analyzing the benefits of RFID technology for cost sharing in construction supply chains: A case study on prefabricated precast components", Automation in Construction, vol. 24, (2012), pp. 120-129.

[22] S. Chae and T. Yoshida, "Application of RFID technology to prevention of collision accident with heavy equipment", Automation in Construction, vol. 19, no. 3, (2010), pp. 368-374.

[23] S. Abdoli, "RFID application in municipal solid waste management system", International Journal of Environmental Research, vol. 3, no. 3, (2009), pp. 447-454.

[24] J. Wyatt, "Maximizing Waste Management Efficiency through the Use of RFID”, Texas Instruments, (2008) April, pp. 1-8.

\section{Authors}

\section{Yanghua Gao}

He received his M.Sc. in Control science and Engineering (2010) from Zhejiang University. Now he is full engineer of informatics at Information Center, China Tobacco Zhejiang Industrial Co.,Ltd. His current research interests include logistics/supply chain management, Radio Frequency Identification and logistics information system.

\section{Zhihua Zhang}

He received his M.Sc. in Computer science technology and Technology (1984) from Zhejiang University. Now he is full associate professor and head of informatics at Information Center, China Tobacco Zhejiang Industrial Co., Ltd. His current research interests include Emergency Response and Stochastic Programming. 